\title{
Typologie morpho-sédimentaire des dépôts actuels de la vallée du Moyen Beht (Sillon sud rifain occidental, Maroc)
}

\author{
Ahmed LAABIDI ${ }^{1}$, Lahcen GOURARI ${ }^{1} \&$ Abdellah EL HMAIDI ${ }^{2}$ \\ ${ }^{1}$ Université Sidi Mohamed Ben Abdellah, Faculté des Sciences, Dhar El Mehraz, Département de Géologie, \\ B.P. 1796, Fès, Maroc.Email : gourari_lahcen@hotmail.fr, laabidi-ahmed@hotmail.fr; \\ ${ }^{2}$ Université Moulay Ismail, Faculté des Sciences de Meknès, Département de Géologie, Equipe Sciences de \\ l'Eau et Ingénierie de l'Environnement, B.P. 11201, Zitoune, Meknès, Maroc. Email : elhmaidiyahoo.fr.
}

\begin{abstract}
Résumé: Le présent travail porte sur l'étude de la sédimentation actuelle de la vallée du Moyen Beht à travers le Sillon sud-rifain occidental, entre sa sortie du Maroc central et le barrage d'El Kansera. Plusieurs milieux morpho-sédimentaires ont été distingués en fonction de la morphologie et de la dynamique sédimentaire. La typologie morpho-sédimentaire de ces unités, leur répartition spatiale et leur développement sont liés étroitement aux conditions morpho-structurales le long de la vallée qui déterminent la typologie des styles fluviaux. Trois tronçons de vallées ont été distingués : deux tronçons à vallées larges, façonnées dans des zones déprimées de type synclinal et composées de terrains tendres argileux et marneux d'âge triasique et miocène, que sépare un tronçon de vallée étroite et fortement incisée dans un dôme anticlinal, formé de terrains durs, carbonatés et d'âge jurassique.

Dans les tronçons où la vallée est large, les styles fluviaux sont méandriformes, avec des méandres libres de plaine. Les dépôts se répartissent en deux types principaux : des dépôts de texture caillouteuse à sableuse et des dépôts de texture silto-argileuse à argileuse et plus au moins riches en matière organique. Les premiers se répartissent dans les fonds de chenaux actifs, les barres, principalement des méandres, et les cônes de déjection. Les seconds occupent les plaines d'inondation, les méandres et les chenaux abandonnés.

Dans le tronçon médian, creusé dans le dôme anticlinal, le style fluvial est de type droit à faiblement sinueux à travers ses deux bordures méridionale et septentrionale et méandriforme avec des méandres de type encaissé à travers sa voûte.

- Le long des bordures, les dépôts de texture caillouteuse à sableuse se répartissent aux fonds des chenaux actifs, dans des barres de type varié (longitudinale, latérale, diagonale et transversale), des levées naturelles et des cônes de déjection alors que les dépôts de texture fine silto-argileuse à argileuse occupent les plaines d'inondation et les chenaux abandonnés.

- le long de la voûte, les dépôts sont de texture caillouteuse à sableuse et occupent les fonds de chenaux, les barres, les banquettes étroites de plaine d'inondation, les cônes de déjection et les bas des versants. Dans ce dernier cas, les dépôts se présentent sous forme de cônes d'éboulis et d'épandages de débris rocheux, détachés des parois et falaises rocheuses taillées dans les roches dures carbonatées d'âge jurassique. Ces dépôts, souvent repris par les ruissellements et la gravité, viennent engraisser les épandages des cônes de déjection.
\end{abstract}

Mots clés: - Dépôts actuels, styles fluviaux, géomorphologie, morpho-structurale, lithostratigraphie, sédimentologie, Moyen Beht, Sillon sud rifain occidental.

\section{Morphosedimentary typology of current deposits of the valley of mean Beht (Furrow south western Rif, Morocco)}

Abstract: - This work focuses on the study of current sedimentation Valley Middle Beht across south western Rif Furrow between the output of central Morocco and El Kansera dam. Several morpho-sedimentary environments were distinguished based on morphology and sediment dynamics. The morpho-sedimentary type of these units, their spatial distribution and development are closely linked to the morpho-structural conditions along the valley that determine the type of river styles. Three sections of valleys were identified: two sections with wide valleys, formed in depressed areas and types of synclinorium composed of soft soil clay and marl of Miocene and Triassic age that separates a stretch of narrow and sharply incised valley in a dome anticlinorium formed of hard surfaces and Jurassic carbonate. In sections where the valley is wide, meandering river styles, with free meandering plain. The deposits are divided into two main types: deposits in sandy and stony texture deposits texture clay to silty clay and more or less rich in organic matter. The first fall in fund assets channels, mainly bars and meandering alluvial fans. The latter occupy floodplains, meanders and abandoned channels. 
In the middle section, dug in Anticlinorium dome, river style is right type weakly meandering through both northern and southern borders and meandering with meandering type cashed through his vault.

- Along the edges, deposits of gravel to sandy texture fall in funds active channel in the bars of various types (longitudinal, lateral, diagonal and transverse), in natural levees and alluvial fans while deposits of finegrained silty clay to clay occupy floodplains and abandoned channels.

- Along the vault, deposits are sandy to pebbly texture and hold funds channels, bars, benches narrow floodplain, alluvial fans and the lower slopes. In the latter case, the deposits are in the form of talus cones and applications of rocky debris detached from the walls and cut in hard rock of Jurassic carbonate rock cliffs. These deposits often picked up by runoff and gravity, just fatten the spreading of alluvial fan.

Keywords: Current deposits, fluvial styles, geomorphology, Morphostructural, lithostratigraphy, sedimentology, Middle Beht, south western Rif Furrow.

\section{INTRODUCTION}

Ce travail a quatre objectifs principaux. Le premier est l'identification des styles fluviaux actuels du lit de l'Oued du Moyen Beht et l'explication de leur formation en relation avec les conditions morpho-structurales le long de la vallée. Le second est la reconnaissance des différentes unités morpho-sédimentaires présentes dans les différents types des tronçons distingués en fonction de la typologie des styles fluviaux. Le troisième est l'examen des relations qui existent entre, d'une part, les unités morpho-sédimentaires strictement fluviatiles du fond de la vallée ou du lit majeur et, d'autre part, les unités liées étroitement à la dynamique des versants dans les tronçons de la vallée distingués.

En fin, le quatrième objectif est la reconstitution des conditions dynamiques des mises en place des différents types de dépôts dans les unités morpho-sédimentaires différenciées en relation avec les conditions environnementales, notamment l'action anthropique, à partir de l'étude morphologique et sédimentologique de ces dépôts.

\section{CONTEXTE PHYSIQUE}

\section{Géomorphologie et géologie}

Le secteur d'étude correspond à la vallée du Moyen Beht qui appartient, de point de vue géographique et géologique, au Sillon sud rifain occidental. Ce secteur s'étend depuis la sortie de la vallée de l'Oued Beht de la Meseta occidentale, en amont, jusqu'au barrage d'El Kansra en aval.

Le bassin hydrographique de l'Oued Beht est un sous bassin de celui de l'Oued Sebou. Les artères hydrographiques de ce cours d'eau prennent naissance dans le Maroc central septentrional et le Moyen-Atlas occidental. Il rejoint l'Oued Sebou inférieur après avoir traversé d'abord le Sillon sud rifain central, où il reçoit les apports d'un certain nombre d'affluents importants, puis le Pré-rif et la partie méridionale de la plaine d'El Gharb qui fait partie du Sillon sud rifain occidental (Fig. 1).

Du point de vue géologique, le secteur d'étude est formé de terrains allant du Paléozoïque jusqu'au Quaternaire [1]. Les formations géologiques du socle paléozoïque et de la couverture s'ennoient, au niveau du Sillon sud rifain, sous des formations d'âge Miocène supérieur). Les terrains miocènes sont surmontés en discordance par les dépôts des terrasses fluviatiles de l'Oued Beht qui sont étagées à emboîtées.

De point de vue structural, le secteur d'étude est affecté par des ondulations d'origine tectonodiapirique comportant deux zones déprimées de type synclinal que sépare une zone en dôme de type anticlinal (Fig. 2). Le réseau de la fracturation est composé principalement par des failles de direction N0 à N20, N40 à N50, N100 à N120 et N140 à N160. Ce réseau a déterminé la configuration du réseau hydrographique actuel (Fig. 2). 


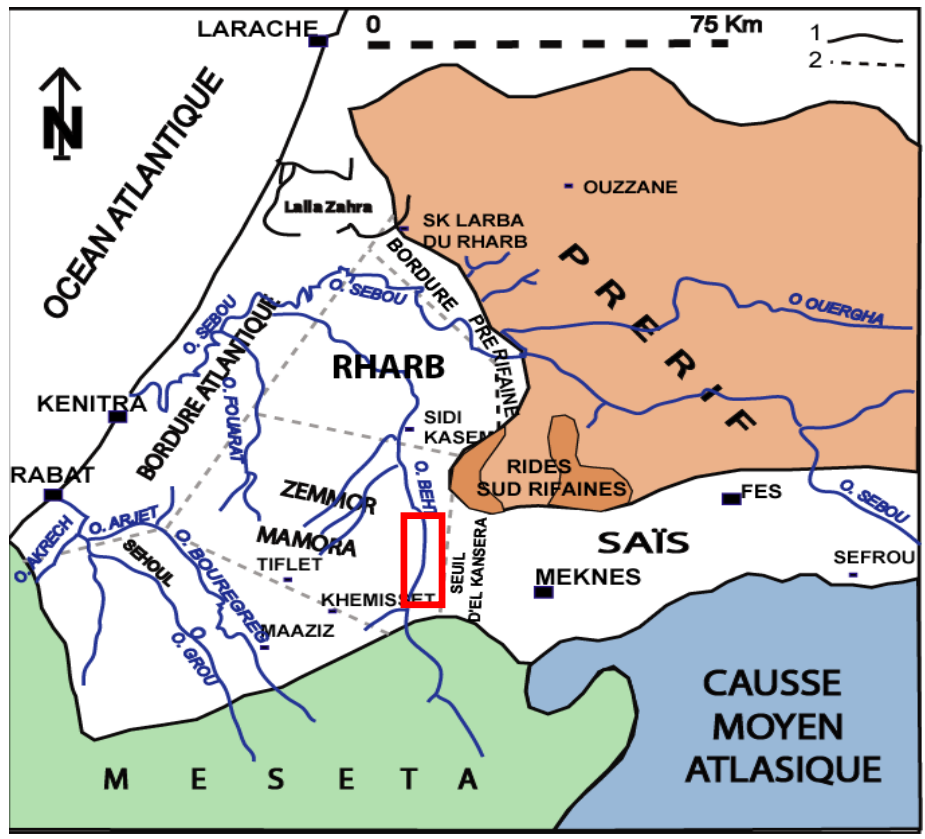

Figure 1. Localisation géographique du secteur d'étude dans son contexte régional [2].

Figure 1. Geographical location of the study area in its regional context [2].

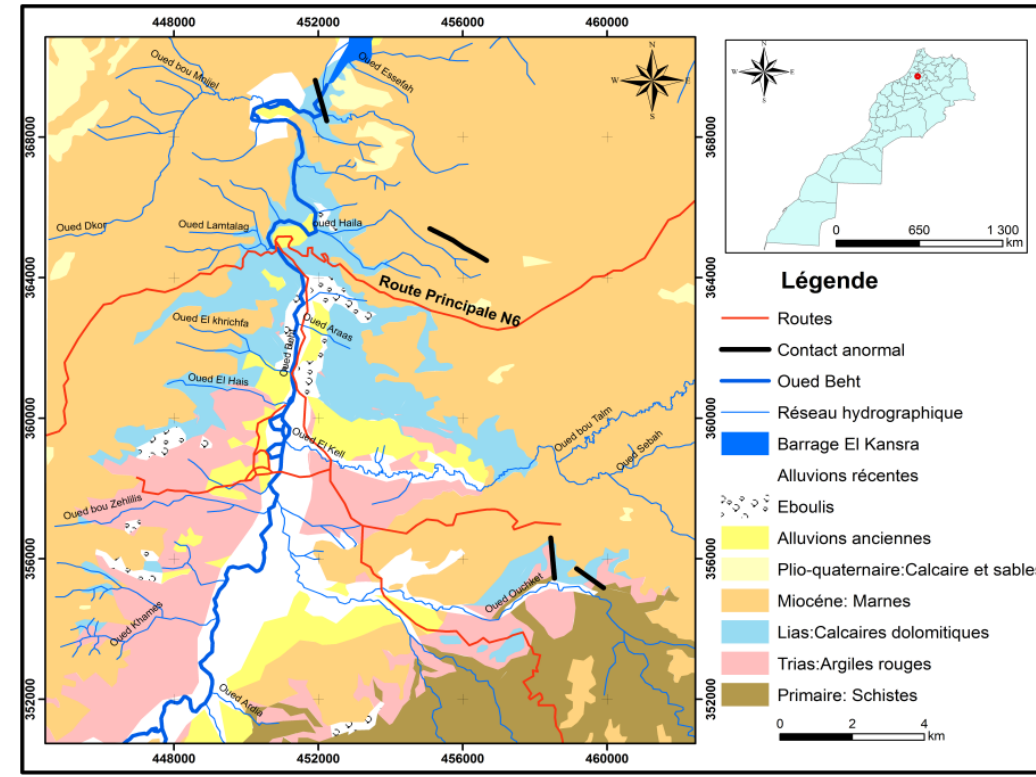

Figure 2. Esquisse géologique du bassin versant du Moyen Beht (d'après [1] la carte géologique de Meknès Nord de 1951 au 1/1000000 modifiée).

Figure 2. Geological sketch Watershed Oued Beht average (after [1] the Geological Map of Meknes North 1951 millionth amended).

\section{Climat et végétation}

Le bassin hydrographique de l'Oued Beht présente un climat de type méditerranéen avec deux saisons : une saison humide et froide et une autre sèche et chaude. Les précipitations moyennes annuelles sont irrégulières avec une moyenne annuelle de $700 \mathrm{~mm}$ sur l'ensemble du bassin. Les pluies sont mal réparties pendant l'année et très irrégulières d'une année à l'autre et d'un mois à l'autre. Ces pluies se font souvent sous forme orageuse et s'expriment par des crues parfois violentes. 


\section{MÉTHODOLOGIE}

L'étude morpho-sédimentaire, lithostratigraphique et sédimentologique a porté sur les dépôts actuels de la vallée du Moyen Beht. Elle a été effectuée grâce à de nombreuses coupes sélectionnées avec soin dans le terrain prospecté. Ces coupes ont été levées, décrites et analysées du point de vue morphologique, lithostratigraphique et sédimentologique. D'autres coupes géologiques ont été levées à travers les formations et les formes de dépôts actuels pour montrer leurs agencements spatiaux et leurs relations géométriques mutuelles.

Cette méthodologie, suivie, a été adoptée dans le but de réaliser une typologie morpho-sédimentaire et de reconstituer la dynamique sédimentaire des formations sédimentaires actuelles et des milieux sédimentaires de leurs mises en place à travers l'étude de la géodynamique des dépôts actuels dont les milieux morphosédimentaires sont encore bien identifiables (cônes de déjection, barres, plaine alluviale, chenaux et bas de versant).

\section{RESULTATS}

\section{Tronçonnage morpho-structural}

Les observations de terrain et l'analyse morpho-structurale de la partie étudiée de la vallée ont permis de la subdiviser en trois grands tronçons (Fig. 3) :

+ Un tronçon supérieur où la vallée est large et creusée dans une zone déprimée de structure synclinale. Cette zone est formée de terrains généralement tendres, composés d'argiles rouges triasiques à intercalation de basaltes doléritiques altérés. Ces terrains sont recouverts en discordance angulaire par des dépôts du Miocène supérieur constitués de conglomérats, de grès friables et de marnes sableuses.

+ Un tronçon moyen ou la vallée est étroite et profonde. Cette vallée est incisée à travers un dôme de structure anticlinale. Ce dôme, d'origine tectono-diapirique, présente une ossature composée de terrains durs carbonatés d'age jurassique qui arment les terrains tendres triasiques. Ces terrains mésozoïques sont couronnés sur les bordures du dôme par les marnes sableuses du Miocène supérieur.

+ Un tronçon inférieur ou la vallée devient de nouveau relativement plus large et moins profonde. Cette vallée est incisée dans une dépression de structure synclinale. Cette dépression est constituée de terrains tendres du Trias et du Miocène supérieur.

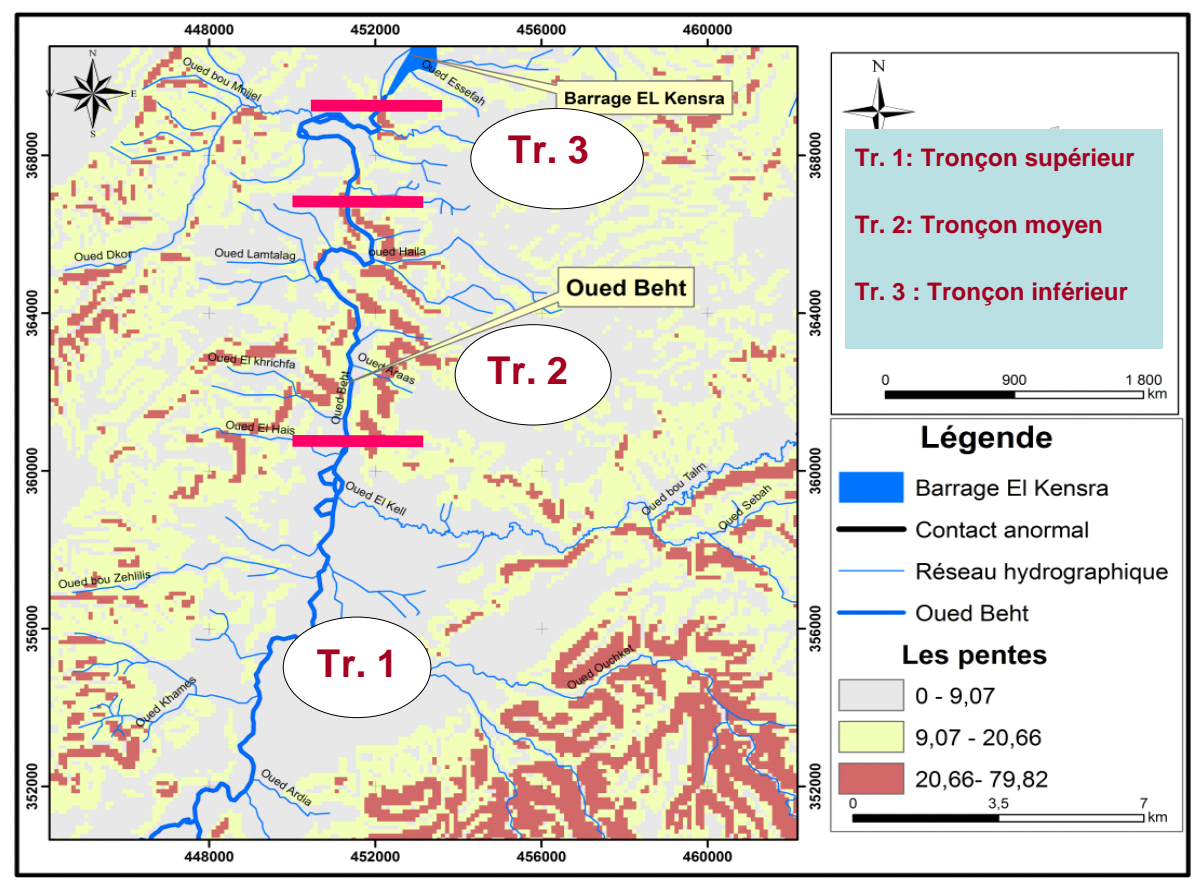

Figure 3 Tronçonnage morpho-structural.

Figure 3 Cutting morpho-structural. 


\section{Typologie des styles fluviaux}

Dans les deux tronçons supérieur et inférieur, les styles fluviaux sont, selon la typologie employée par [3] et [4], sont de type méandriforme, avec des méandres libres ou de plaine et une tendance locale au tressage. Ce style méandriforme devient droit à faiblement sinueux à travers le flanc méridional et le flanc septentrional du dôme anticlinal et méandriforme avec des méandres encaissés à travers sa voûte (Fig. 4).
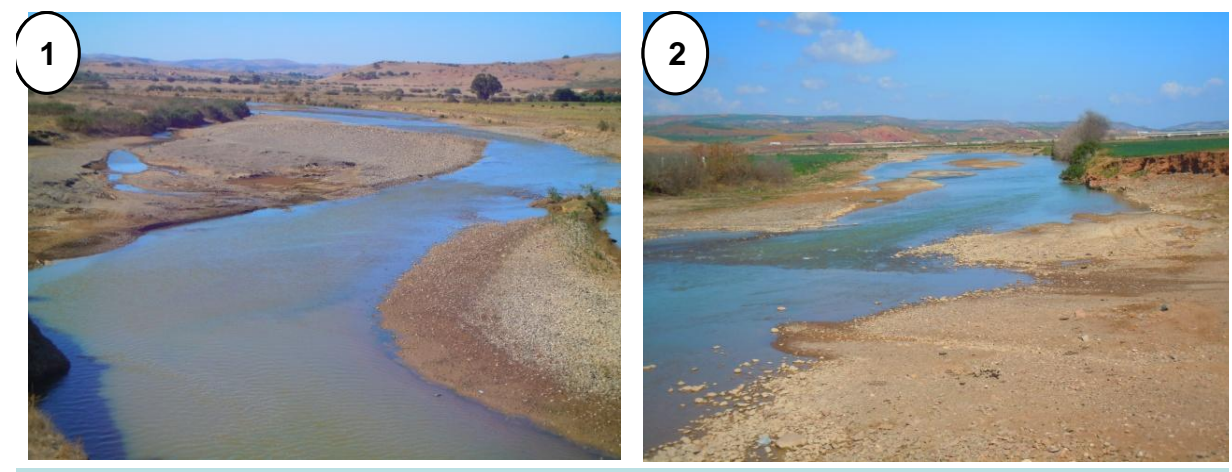

Méandres libres du tronçon supérieur (1) et tendance au tressage (2)

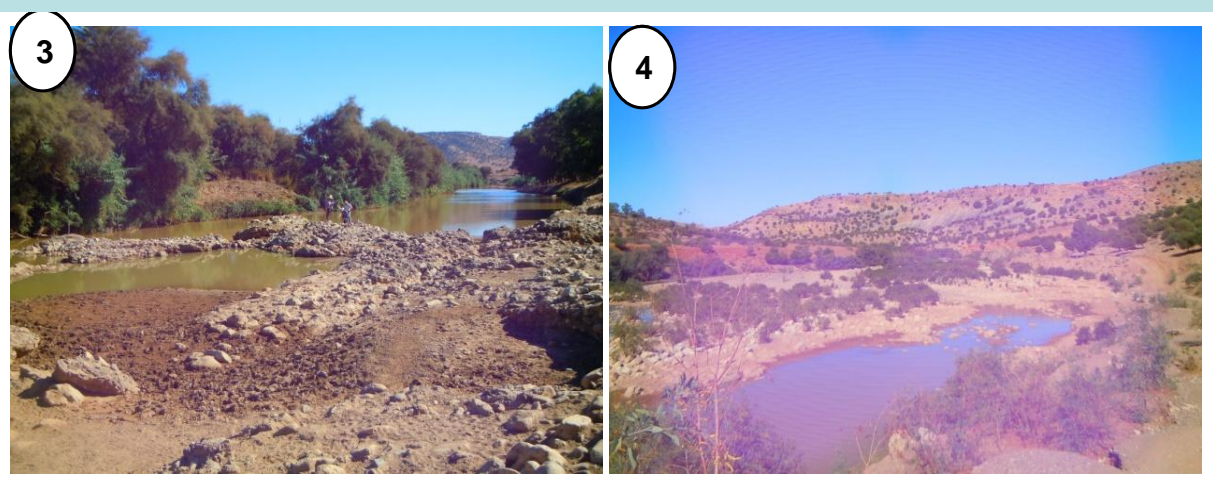

Style faiblement sinueux (3) et méandres encaissés (4) du tronçon médian

Figure 4. Typologie des styles fluviaux dans le secteur d'étude.

Figure 4. Typology of fluvial styles of the study area.

\section{Typologie des milieux morpho-sédimentaires}

Les types des milieux morpho-sédimentaires, leur développement et leur répartition, le long du fond de la vallée du Moyen Beht, dépendent d'un certains nombre de facteurs pouvant être interdépendants. Parmi ces facteurs, on a le style fluvial que peut prendre le lit mineur le long de la vallée, la largeur de celle-ci, sa morphologie, la lithologie de ses versants, la répartition spatiale et l'importance de ses artères hydrographiques latérales affluentes, la présence et l'absence des ceintures végétales le long des berges et au fond du lit mineur etc. Parmi ces facteurs, les plus importants sont les types des styles fluviaux qui peuvent s'enchaîner le long du lit fluvial et qui dépendent, entre autres, des conditions morpho-structurales et de la pente longitudinale de ce lit.

Les observations de terrain, le long du secteur étudié, ont permis de distinguer diverses unités morphosédimentaires. Il s'agit de chenaux actifs, de barres, de levées naturelles, de plaine d'inondation, de chenaux et de méandres abandonnés, de cônes de déjection, de cônes d'éboulis et d'épandages de pente (Fig. 5). 


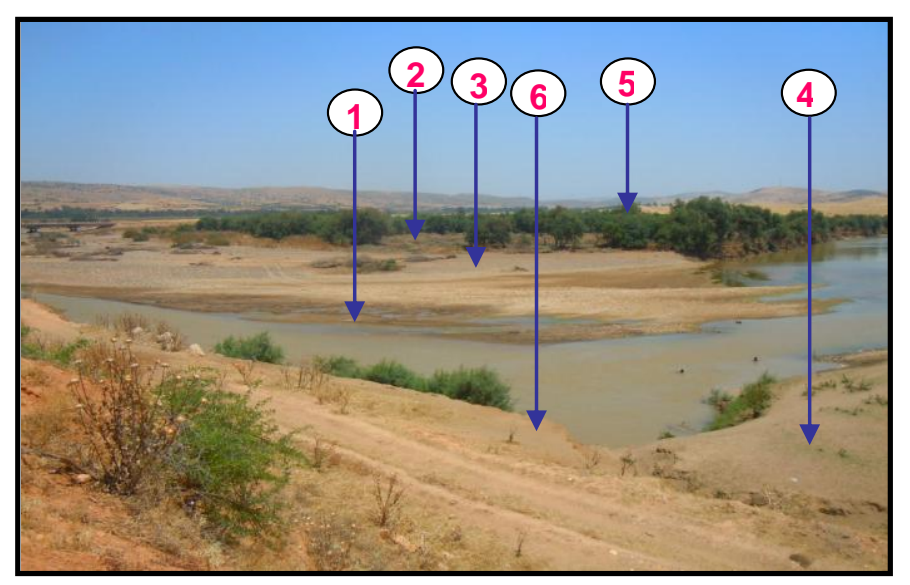

Figure 5. Milieux morpho-sédimentaires de dépôts. 1: Chenal actif, 2 et 3: barre de méandre chenalisée ave en (2) barre de méandre supérieur et en (3) barre de méandre inférieur, 4: méandre abandonné, 5: plaine d'inondation très étendue et colonisée par une végétation naturelle de Tamarix, 6: petite banquette de plaine d'inondation épargnée par l'érosion qui se fait par sapement au niveau de la rive concave.

Figure 5. Backgrounds morpho-sedimentary deposits. 1: Channel active, 2 and 3: Channalised meander bar with in (2): higher bar meander and in (3): lower bar meander, 4: meander abandoned 5: plain extensive flooding and colonized by natural vegetation of Tamarix, 6: Small bench floodplain untouched by erosion is by undercutting at the concave side.

\subsection{Chenaux actifs}

Ces chenaux sont de deux types : principaux et secondaires. Les chenaux principaux sont au nombre de un dans les parties méandriformes du lit mineur et de deux à trois aux niveaux des parties où le lit mineur présente une tendance à être tressé ou droit à faiblement sinueux. Cette tendance au changement du style est liée au rétrécissement du lit mineur et /ou à une accentuation de sa pente longitudinale. Ces chenaux sont limités par des barres différentes où des parties d'une même barre qui est souvent de type longitudinal et morcelée par des chenaux. Les chenaux secondaires correspondent souvent à des chenaux principaux qui ont été délaissés par les écoulements longitudinaux après une migration latérale du lit mineur. Cette migration, qui est due à un encombrement des chenaux par alluvionnement, s'effectue en périodes des décrues lorsque les eaux fluviales s'amoindrissent et se concentrent dans les chenaux principaux, creusés dans les zones les plus basses du lit mineur.

\subsection{Barres}

Ces unités morpho-sédimentaires, qui sont émergées pendant les périodes des basses eaux et pouvant être végétalisées ou non, sont d'extension métrique à décamétrique et présentent des formes et des positions différentes dans le lit mineur. Les formes les plus dominantes sont les formes lenticulaires, en croissant et en linguoides.

\subsection{Levées naturelles}

Ces levées se présentent, le long de certains tronçons des berges, sous forme de cordons dissymétriques de 5 à 10m de largeur. Elles présentent un flanc court et abrupt du côté du lit mineur et un autre plus long et moins abrupt du côté de la plaine d'inondation. Ces milieux morpho-sédimentaires de berges sont colonisés localement par une végétation bien développée de Tamarix. Elles se développent surtout le long des tronçons droits à faiblement sinueux des lits fluviaux mais on les trouve également le long des tronçons méandriformes, surtout au niveau des rives concaves des méandres où elles sont relativement moins développées. Il semble que la formation de ces unités morpho-sédimentaire est liée à une installation d'une végétation d'arbustes qui joue le rôle d'un filtre permettant le piégeage et le dépôts par aggradation de la charge sédimentaire sableuse en périodes de débordement des eaux les plus importantes. 


\subsection{Plaine d'inondation}

Elle se présente sous forme de topographies planes de part et d'autre du lit mineur de l'oued. Cette plaine prend une extension importante dans le tronçon supérieur et le tronçon inférieur du secteur étudié où l'oued développe des méandres libres, parfois de grand rayon de courbure. Le long de ces deux tronçons, les banquettes de cette plaine surplombent le lit mineur par une rupture de pente de longueur et de hauteur différentes. Cette rupture de pente, longue et faible du côté des rives convexes, devient relativement plus courte, plus abrupte et présente une hauteur de 1,5 à $3 \mathrm{~m}$ environ au niveau des rives concaves les plus encaissées. Ces banquettes deviennent relativement beaucoup moins étendues dans le tronçon médian où elles se présentent, de part et d'autre du lit mineur, sous forme de banquettes parfois très étroites. Dans ce tronçon l'érosion de ces banquettes se fait vigoureusement par sapement des berges, surtout de celles qui ne sont pas stabilisées par des ceintures végétales de tamarix.

\subsection{Méandres abandonnées}

Ces milieux morpho-sédimentaires se répartissent principalement dans les tronçons supérieur et inférieur qui disposent d'une large plaine alluviale et de méandres bien développés. Les méandres abandonnés sont reconnaissables sur le terrain par leurs tracés en forme de pédoncules ou de boucles déprimées, étroites, allongées et limitées par deux petites berges qui deviennent de moins en moins exprimées avec le comblement par alluvionnement. Ces boucles peuvent être matérialisées par le développement d'une ceinture végétale de tamarix. L'observation, de prés de ces méandres abandonnés, montre que les dépôts ultimes sont composés de silts argileux affectés par un réseau de fentes de dessiccation bien développées et de forme polygonale. La formation de ces polygones de grande dimension est l'indice d'une importante sédimentation fine qui s'est produite dans des plans d'eau temporaires ayant subi une intense évaporation. Des observations complémentaires ont montré que ces polygones, délimités par des fentes de dessiccation profondes, se transforment en boules juxtaposées. Ces boules résultent d'un affouillement et d'une altération par les écoulements drainés par des chenaux en périodes des crues. Dans ces méandres, qui ne sont pas complètement abandonnés, peuvent stagner des eaux sous forme de flaques d'étendue variée en fin des crues.

\subsection{Cônes de déjection}

Ils existent dans les trois tronçons de la vallée du Moyen Beht où ils se développent davantage dans les deux tronçons supérieur et médian. L'édification de ces unités morpho-sédimentaires est assurée par des artères hydrographiques qui débouchent directement au fond de la vallée et qui proviennent des versants de la vallée ou des régions lointaines, situées dans le Moyen Atlas occidental et le Maroc central oriental. L'étendue de ces cônes dépend étroitement de l'importance des artères hydrographiques latérales et de leurs bassins versants ([5], [6] et [7]). Les épandages des cônes de déjection, édifiés par les artères hydrographiques latérales importantes, se raccordent vers l'amont à des dépôts de divers types morphologiques des fonds des vallées affluentes : chenaux, barres de type varié et plaine d'inondation. Différents types ont été distingués : les cônes édifiés dans le lit mineur, les cônes édifiés aux pieds des versants et les cônes édifiés sur les replats de la plaine d'inondation ou qui y viennent s'emboîter par l'intermédiaire d'une surface de ravinement importante.

\subsection{Cônes d'éboulis et épandages de pente}

Ils se répartissent principalement dans le tronçon médian où leur existence est étroitement conditionnée par la présence de falaises ou de talus rocheux taillés dans les roches dures carbonatées du Jurassique. Ces types de dépôts se développent principalement sur le versant droit de la vallée, à exposition Ouest, et les versants des vallées secondaires latérales qui le sillonnent. Le remaniement de ces dépôts et leurs évacuations par les eaux de ruissellement et d'écoulements torrentiels, que drainent les ravins et les vallées latérales, et qui sont assistés par la gravité, contribuent à l'approvisionnement et à l'engraissement des épandages des cônes de déjection et des alluvions du fond de la vallée en décharges détritiques. 


\section{Types des dépôts et dynamiques de mises en place \\ 4.1. Dépôts des fonds des chenaux}

Les dépôts des fonds des chenaux principaux sont composés de galets et graviers à blocs plus au moins abondants. Ceux-ci, de diamètre décimétrique, sont abondants dans les dépôts des fonds de chenaux du lit mineur du tronçon médian où la torrentialité des écoulements est importante en périodes des crues. Par contre, ils sont rares dans ceux des deux tronçons supérieur et inférieur où ils sont repris à partir des dépôts des cônes de déjection, édifiés en contact du lit mineur. Ces éléments grossiers, qui constituent la charge de fond qui est transportée par roulement et/ou charriage, se présentent sous forme de pavages. Ils peuvent présenter une texture jointive ou non et sont souvent associés à des sables plus au moins graveleux remplissant les interstices entre les galets et les graviers. Ces derniers éléments, de forme sub-anguleuse à sub-arrondie et de natures pétrographiques variées, peuvent être disposés en vrac ou montrer une imbrication donnant un sens de courant conforme au sens du courant actuel. Les dépôts sont, composés de cailloutis hétérométriques. Cette hétérométrie, beaucoup plus importante dans les chenaux des lits droits et des méandres encaissés, est liée à une mise en place par des courants turbulents de type torrentiel et à turbulence bien contrastée au cours du dépôt.

Les chenaux secondaires, qui se présentent à sec ou qui ne drainent que des filets d'eau de très faible vitesse en périodes d'étiage, selon leur position dans le lit mineur et leur altitude par rapport au chenal principal qui suit les zones les plus bases, présentent des remplissages fins composés de sables et de silts argileux. Ces remplissages, qui couvrent des dépôts de cailloutis constitués de galets et graviers à matrice sableuse à sablograveleuse, montrent des rides de courant et des bioturbations et contiennent souvent des débris de matière organique. Dans les flaques d'eau, alimentées par ces filets d'eau, les dépôts fins deviennent plus abondants et s'enrichissent vers le sommet en particules fines et en matière organique. Les parties exondées de ces dépôts montrent un litage fin et sont affectées par des fentes de dessiccation et des traces de gouttes de pluie. Elles montrent également des bioturbations dont les plus importantes sont des traces de racines, de locomotions de vers, de gastéropodes et de pattes d'oiseau.

Les dépôts relativement anciens se caractérisent, au niveau des coupes transversales, par leur formes en gouttière symétrique ou asymétrique et d'extension souvent métrique et à base ravinante, leur texture conglomératique ou caillouteuse, leur hétérométrie plus au moins exprimée, et leur stratification arquée et entrecroisée plus au moins nette et de grande échelle.

\subsection{Dépôts des barres}

Ils sont constitués de cailloutis et sables grossiers à moyens plus au moins graveleux. Ces dépôts, pouvant être chenalisés, sont caractérisés par leur type de progradation. Cette progradation, qui s'accompagne aussi d'une aggradation des dépôts, est latérale par rapport à l'axe du chenal dans le cas des barres de méandres, parallèle à celui-ci dans le cas des barres longitudinales, latérales, transversales et diagonales. Cette progradation des dépôts est induite par une pente. Cette dernière est latérale dans le cas des barres des méandres et liée à une migration du chenal par recul de la rive concave par incision et sapement. Elle est longitudinale dans les autres barres et assure leur déplacement vers l'aval. Les observations effectuées aux niveaux de ces barres montrent que les dépôts sommitaux qui occupent les zones les plus hautes des barres sont lavés, de texture jointive et présentent les éléments les plus grossiers. Ceci est lié à la forte turbulence des écoulements de crues qui provoquent un vannage des galets et des graviers et une évacuation des fines sur les bordures ou les flancs des barres lors des retraits des eaux aux débuts des décrues.

Parmi ces barres, les plus typiques sont les barres des méandres libres les plus développées qui se répartissent dans le deux tronçons supérieur et inférieur du secteur de l'Oued Beht moyen. Ces barres sont caractérisées par leur forme hémisphérique ou en croissant et leur position aux niveaux des rives convexes des méandres. Dans certains rares cas, ces unités morpho-sédimentaires peuvent être de forme lenticulaire allongée et avoir un grand axe longitudinal et un grand axe transversal qui ont des longueurs qui ne présentent pas de grande différence comme dans le cas des vraies barres longitudinales et linguoides où cette différence est grande. Ces barres de méandre de forme lenticulaire sont bordées par deux chenaux curvilignes de configurations opposées : un chenal principal et un autre secondaire en voie de délaissement. Ceci montre que cette forme est liée à la juxtaposion de deux barres en partie superposées et dont les progradations se sont faites dans deux sens opposés. La zone d'emboîtement ou de superposition de ces deux barres se présente en dôme et correspond à l'endroit où le dépôt est le plus épais. 
Ces barres sont très sollicitées par les carriers pour l'extraction des sables. L'observation et l'analyse sédimentologique des petits fronts de carrières et d'excavation d'extraction des sables montrent que ces dépôts sont formés d'un mélange de sables et de cailloutis qui s'organisent en couches obliques et progradantes. Ces dépôts d'enrichissent en sables vers le haut des barres et présentent des figures de chenalisation et des surfaces de ravinement fréquentes limitant des séquences sédimentaires. Les dépôts de sables, allant depuis les sables graveleux aux sables fins, s'organisent en couches obliques de 10 à $40 \mathrm{~cm}$ d'épaisseur. Ces couches présentent une stratification parfaite, continue, plane, oblique et montrent des discordances liées aux différences de pendages sédimentaires des couches qui diminuent vers le haut simultanément à une décroissance du diamètre des grains et des épaisseurs des couches. Dans certains niveaux, le litage sédimentaire ou la stratification devient diffuse ou disparaît complètement à cause de sa perturbation par des réseaux racinaires.

Les caractères sédimentologiques de ces barres de méandres libres de plaine des deux tronçons supérieur et inférieur disparaissent en grande partie dans les barres des méandres encaissés du tronçon médian. Ici, les dépôts montrent une forte hétérométrie des cailloutis qui renferment des blocs de diamètre décimétrique en grande quantité et qui sont disposés en vrac. Cette différence de caractères sédimentologique, entre les dépôts des barres des méandres libres et celles des méandres encaissés, est liée étroitement à des dynamiques de mises en place différentes. Ces dynamiques sont de type fluviatile dans le premier cas et de type torrentiel dans le second (Fig. 6).

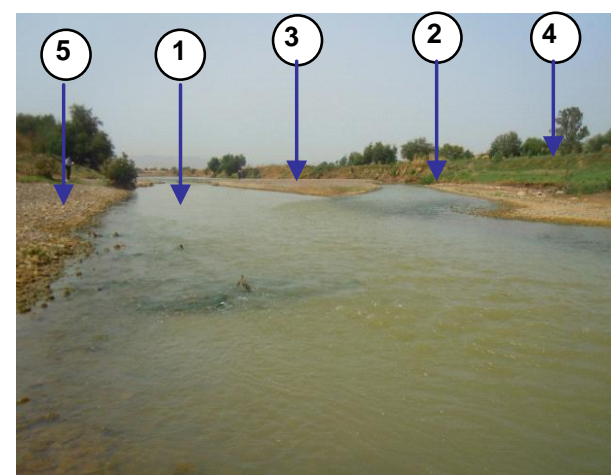

Deux chenaux 1 et 2 séparés par une barre longitudinale 3 et délimités par deux barres de rive ou latérales 4 et 5 .

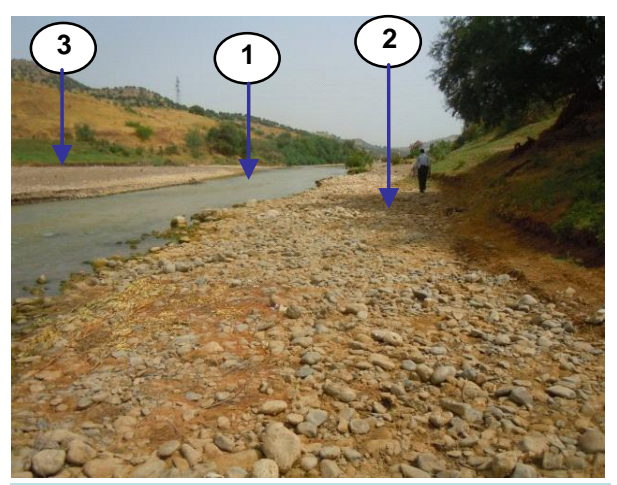

Chenal 1 délimité par une barre de rive ou latérale 2 et une barre longitudinale 3 .

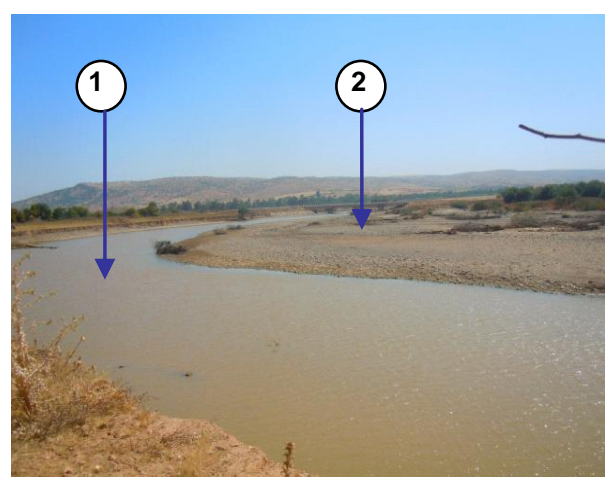

Chenal 1 et barre de méandre 2

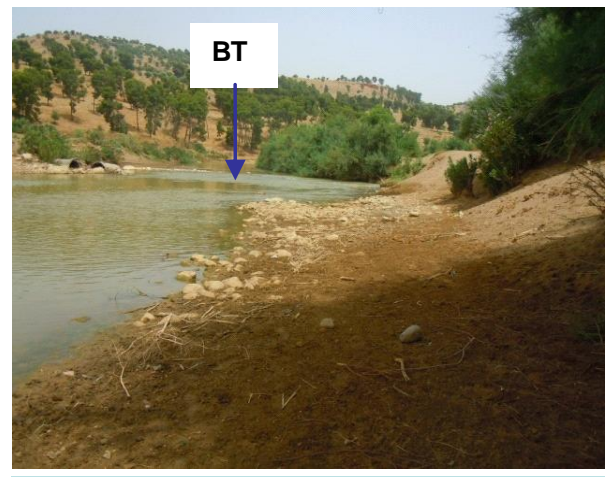

Barre transversale progradante vers l'aval et formant un seuil séparant deux mouilles où l'écoulement est relativement plus calme.

Figure 6. Dépôts de fonds de chenaux principaux et de barres.

Figure 6. Deposits of funds of main channels and of bars.

\subsection{Dépôts des méandres abandonnés}


Ces dépôts, qui sont reconnaissables en surface par leur morphologie en boucle et en coupe transversale par leur morphologie en gouttière, débutent par des cailloutis et des sables à stratifications incurvées et entrecroisées et organisés en séquences élémentaires strato-grano-décroissantes. Ces dépôts, de chenaux ou de méandres actifs, sont surmontés par des sables fins et des silts argileux. Ces sédiments, qui constituent les remplissages des méandres après leurs abandons définitifs sont organisés en séquences élémentaires positives et répétitives. Les bases de ces séquences présentent des surfaces de ravinement plus au moins exprimées. Les dépôts débutent par des sables moyens à stratifications obliques incurvées à planes et entrecroisées puis se suivent vers le haut par des sables fins à stratifications ondulées à planes et horizontales que coiffent des silts argileux finement lités et localement riche en matière organique et en sels diffus de couleur blanchâtre. Les niveaux sableux présentent des couleurs claires pouvant être grisâtres ou beiges alors que les niveaux de silts argileux ont des couleurs sombres allant du brun foncé au noirâtre (Fig. 7).

Ces séquences de dépôts de comblements répétitives, complètes ou tronquées, sont mises en place lors de l'immersion des bras morts par les eaux de débordement en provenance du lit mineur et de ruissellement à partir des versant lors des périodes de crues importantes. Les premiers écoulements qui arrivent dans les bras morts, de manière diffuse à chenaillée, entraînent le dépôt des sables des bases des séquences. Les autres niveaux, plus fins, qui viennent au dessus, sont mis en place par décantation dans le plan d'eau retenu dans la zone déprimée du méandre abandonné qui se comporte alors comme un lac éphémère. La matière organique associée aux sédiments, qui est responsable de leur coloration noirâtre, peut être autochtone et/ou allochtone. Les dépôts de sels, diffus ou légèrement concentrés dans ces dépôts fins, résultent d'une précipitation physicochimique des sels en solution, solubilisés à partir des argiles salifères triasiques, par évaporation.

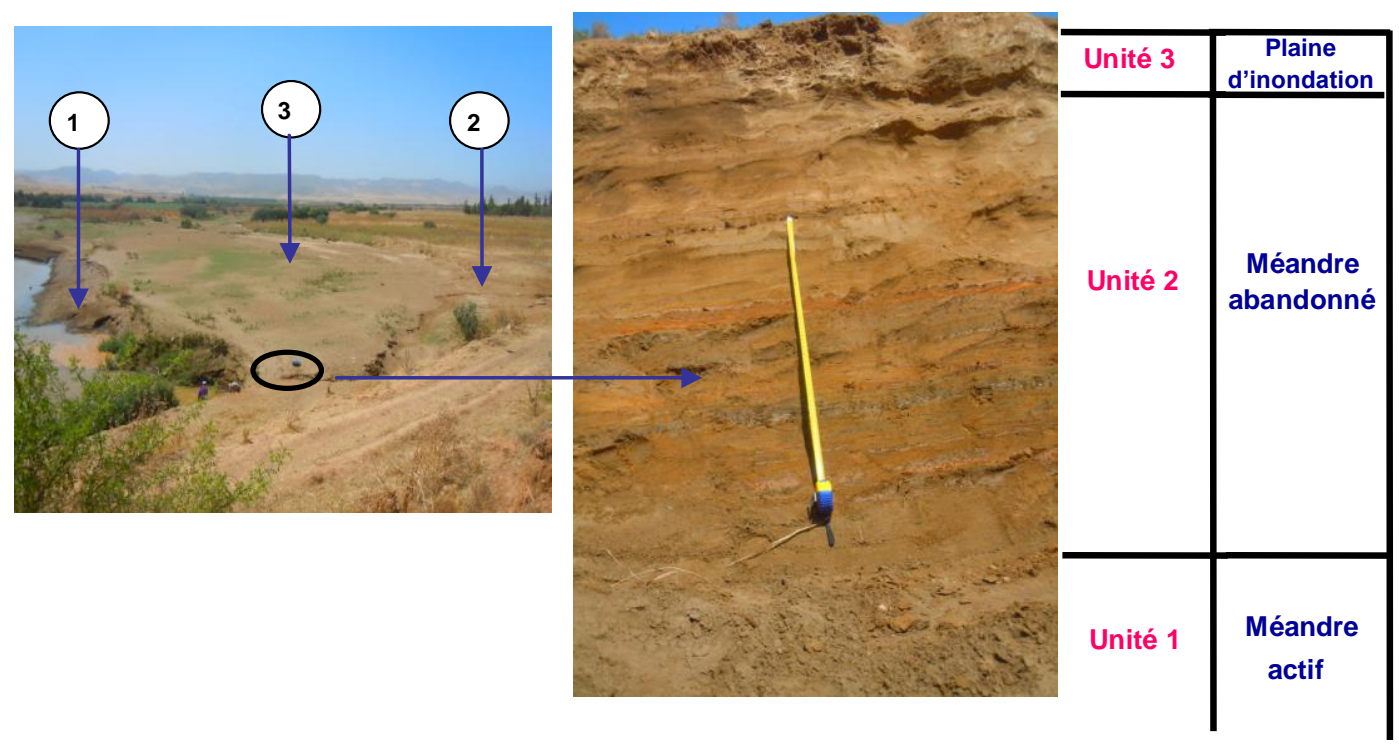

Figure 7. Coupe dans les dépôts de comblement du méandre abandonné (2). Unité 3: sables moyens à fins à stratification oblique et entrecroisée à la base et horizontales au sommet et inter lits de silts argileux brunâtres et intercalations de lentilles de sables grossiers graveleux et chenalisés. Unité 2: sables moyens à fins à base souvent ravinante de couleur beige à rosâtre et à stratification variée : oblique plane à arquée, ondulée et horizontale et silts argileux et argiles brunâtres, verdâtres, noirâtres, finement lités, à traces de racines, inter-lits de matière organique, fragments de charbon de bois et dépôts de sels en lits lenticulaires minces ou diffus. Unité 1: cailloutis à matrice sableuse et à stratification arquée de grande échelle et à intercalations sableuses.

Figure 7. Cup in deposits of the filling of the abandoned meander (2). Unit 3: Sand means for oblique stratification and crisscross at the base and at the top and horizontal inter beds of brownish clayey silts and interbedded lenses of coarse sand and gravel channelized. Unit 2: Sand to ends often based ravinant beige to pinkish varied stratification oblique planar curved, wavy, horizontal and silts and clays clay brownish, greenish, blackish, finely bedded, with traces of roots, inter beds organic material, fragments of charcoal and salt deposits thin diffuse or lenticular beds. Unit 1: Crushed to sandy matrix and arched stratification scale and sandy intercalations

\subsection{Dépôts des levées naturelles}


Les dépôts des levées naturelles sont formés essentiellement de sables moyens à stratification oblique et entrecroisée, de petite échelle et incurvées à planes, que surmontent d'abord des sables fins à stratification plane et horizontale puis des silts argileux à litage horizontale (Fig. 8). Ces dépôts sont affectés par une forte bioturbation des réseaux racinaires de la ceinture végétale arbustive composée principalement de tamarix. Cette ceinture végétale joue deux rôles importants: elle permet le piégeage des sédiments en période des débordements des hautes eaux, lors des crues les plus importantes, et protègent ces levées contre l'érosion par les eaux courantes et le vent.

La mise en place des dépôts des levées naturelles est assurée par les eaux de débordement du lit mineur lors des crues. L'édification de ces levées, actuellement surélevées d'environ 2 à $3 \mathrm{~m}$ par rapport au niveau d'étiage, s'est faite de manière progressive par des inondations de plus en plus importantes, déclenchées par des crues de plus en plus amples et violentes. Les premiers dépôts sont mis en place au début de l'inondation par les premières eaux de débordement qui présentent une turbulence plus au moins importante permettant le transport d'une charge solide en suspension à travers les levées vers la plaine d'inondation. Une grande partie de la fraction sableuse grossière à moyenne de cette charge se dépose au niveau des levées par des écoulements suffisamment turbulents pour engendrer des rides de courant dissymétriques par des courants d'eau de directions différentes et qui se font vers la plaine d'inondation. Ces rides s'expriment en coupe par des stratifications obliques et entrecroisées d'abord arquées puis planes. Lors du maximum de l'inondation, qui touche aussi bien les levées que la plaine d'inondation, la turbulence des eaux baisse et permet la décantation des sables fins et des silts argileux qui continuent à s’y faire en période des décrues.
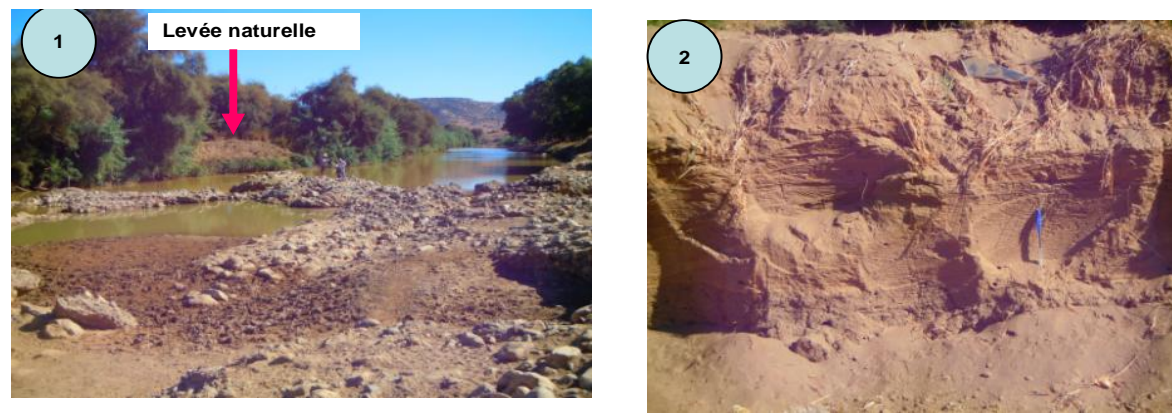

Figure 8. Dépôt des levées naturelles.

Figure 8. Deposit of natural levees.

\subsection{Dépôts de plaine d'inondation}

Les observations de terrain le long du secteur d'étude ont montré que les coupes les plus importantes sont situées aux niveaux des talus des rives concaves des méandres. Ces coupes, épaisses de 2 à $3 \mathrm{~m}$ environ, comportent généralement deux niveaux sédimentaires : un niveau de texture conglomératique ou caillouteuse à la base et un autre de texture silto-sablo-argileuse au sommet. Les dépôts de texture conglomératique correspondent à des dépôts de fond de lit mineur. Ils ont été mis en place par des écoulements turbulents dans des chenaux et des barres. Les dépôts de texture fine correspondent à des dépôts de plaine d'inondation. Leurs mises en place ont été principalement faites par décantation lors des débordements des eaux dans le lit majeur. Les témoins de cette décantation sont les litages fins et horizontaux des couches de texture silto-argileuse. La présence de niveaux lenticulaires, sableux à sablo-graveleux voire même conglomératiques, témoigne de mises en place par des écoulements suffisamment agités aux débuts ou en fin des inondations lorsque la profondeur des eaux devient faible et celles-ci s'écoulent de manière diffuse ou concentrée dans des chenaux généralement peu profonds et larges.

La richesse de ces dépôts en fentes de dessiccation exprime leurs exondations fréquentes et prolongées. Les indices de ces exondations sont la richesse de ces dépôts en traces de racines et la présence de plusieurs intercalations de paléosols peu évolués (Fig. 9). 


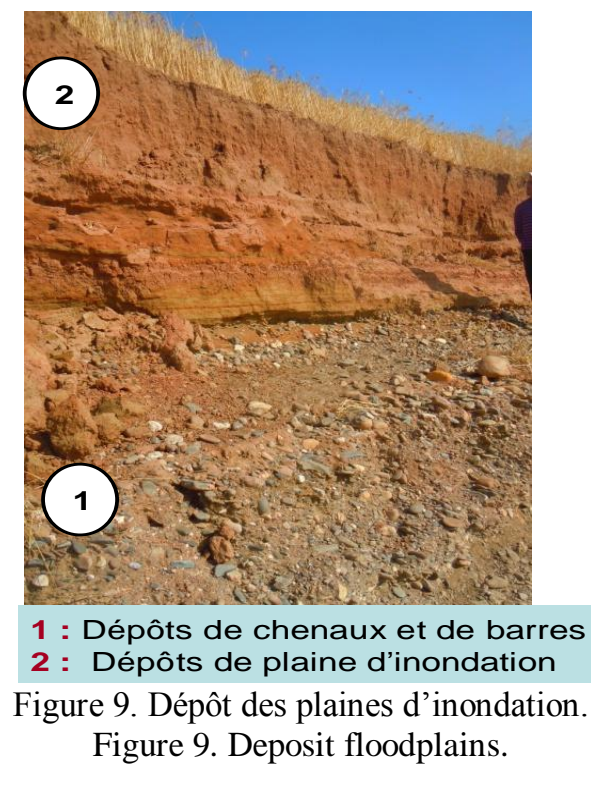

\subsection{Dépôts des cônes de déjection}

Ces dépôts sont constitués de cailloutis hétérométriques à blocs lavés ou pris dans une matrice siltoargileuse plus au moins abondante (débris flow d'après [8]) et de silts argileux avec ou sans galets et graviers épars (mud flow d'après [8]). Ils sont disposés en couches horizontales ou progradantes et organisés en séquences élémentaires strato-grano-décroissantes (Fig. 10).

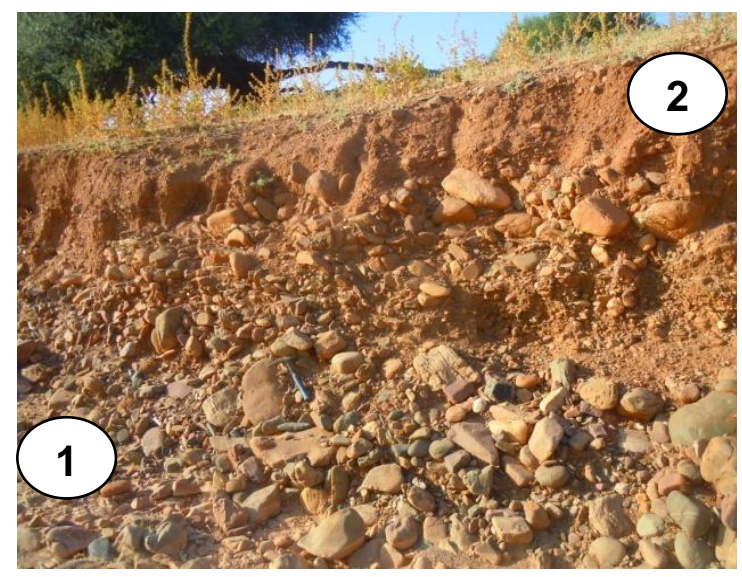

Figure 10. Dépôts des cônes de déjection. 1: Débris flow et 2: Mud flow.

Figure 10. Deposits of alluvial fans. 1: Debris flow and 2: Mud flow.

Les observations de terrain effectuées, surtout au fond de la vallée et le long des berges du lit mineur, ont montré que les épandages des cônes de déjection, édifiés par les artères hydrographiques latérales, aux niveaux de leurs confluences avec le lit mineur de l'Oued Beht moyen, se répartissent en deux voire même trois générations emboîtées. Les cônes de la génération la plus ancienne qui sont les plus étendus, s'emboîtent dans les dépôts de la plaine d'inondation par l'intermédiaire d'une surface de ravinement importante. Les épandages des cônes de la génération la plus jeune sont les moins étendus et peuvent se présenter en forme de pattes d'oiseau et passent latéralement aux dépôts des fonds des chenaux et des barres. Les épandages des cônes de la génération intermédiaire, supportent des dépôts sableux bien structurés. Ces dépôts, éolisés en surface, sont composés de sables moyens à fins à stratification d'abord oblique, incurvée à plane et entrecroisée puis horizontale que surmontent des silts argileux à litage horizontal. Ces dépôts, qui ont été mis en place lors des périodes des débordements des eaux du lit mineur de l'Oued Beht moyen et du cône de déjection, seraient d'origine mixte. Cette banquette de plaine, qui est surplombée par la plaine étendue de l'Oued Beht, constitue un exemple de dédoublement de la plaine d'inondation actuelle aux niveaux des zones de confluence. 


\subsection{Dépôts de pente}

Ils se répartissent principalement dans le tronçon médian et se présentent sous forme de cônes d'éboulis et d'épandages de débris rocheux détachés des parois et falaises rocheuses taillées dans les roches dures carbonatées d'âge jurassique. Ces dépôts, souvent repris par les ruissellements et la gravité, viennent engraisser les épandages des cônes de déjection (Fig. 11).

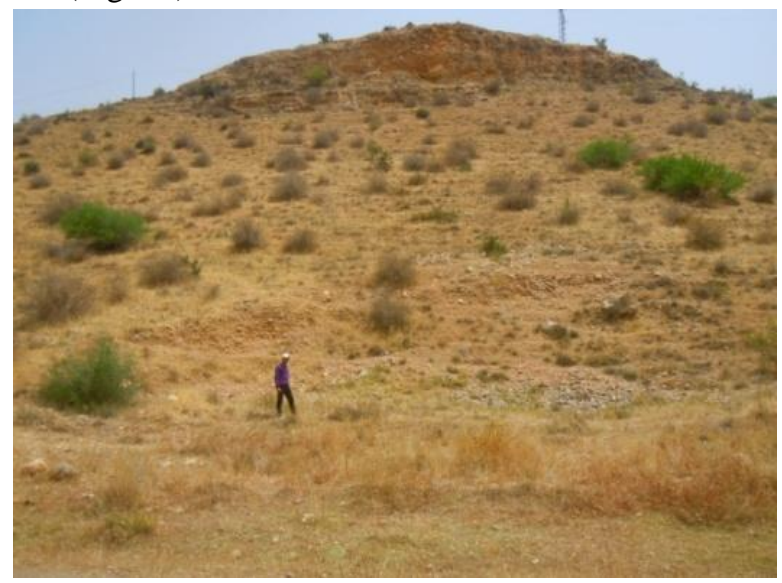

Figure 11. Dépôts de pente.

Figure11. Slope deposits.

\section{DISCUSSION}

1. Conditions environnementales de mises en place des dépôts

L'étude menée sur les dépôts du fond de la vallée actuelle a permis de distinguer plusieurs types morphologiques qui peuvent être répartis en deux principaux types : les dépôts des versants et les dépôts du fond de la vallée ou du lit majeur.

Les dépôts, liés à la dynamique des versants de la vallée, sont de deux types morphologiques : les dépôts de pente ou des bas des versants et les dépôts des cônes de déjection. Les premiers se sont mis en place lors des éboulements des parois rocheuses carbonatées, fragilisées par la gélifraction et l'élargissement des fissures et des fractures par dissolution karstique. Les seconds sont mis en place par des écoulements fluviatiles torrentiels, intermittents et très chargés en produits détritiques en provenance de sous bassins versants dénudés ou à très faible couvert végétal. Ces écoulements sont déclenchés par des pluies souvent de caractère orageux.

Les dépôts du lit majeur, mis en place principalement par les écoulements longitudinaux du lit du Moyen Beht, sont de types morphologiques variés selon le type du style fluvial qui est étroitement lié aux conditions morpho-structurales le long de la vallée. En effet, ces conditions, qui jouent un rôle prépondérant dans la détermination des pentes en long des cours d'eau, leurs débits et leurs charges sédimentaires, déterminent le type du style fluvial dans un tronçon donné du lit du cours d'eau. Les différents types morphologiques qui ont été distingués peuvent être répartis en trois types principaux : les dépôts du lit mineur, les dépôts des berges et les dépôts de plaine d'inondation. Les dépôts du lit mineur comportent les dépôts des chenaux actifs principaux et secondaires, les dépôts des barres et les dépôts des chenaux et des méandres en voie de délaissement. Les dépôts des berges correspondent aux dépôts des levées naturelles. Les dépôts de plaine d'inondation comportent les dépôts de plaine d'inondation au sens strict et ceux des méandres abandonnés. Exceptés les quelques passées de matière organiques et salifères, rencontrées dans les dépôts des chenaux et des méandres abandonnés, tous les autres dépôts sont formés de faciès détritiques allant depuis des silts argileux avec ou sans galets et graviers épars aux cailloutis à blocs avec ou sans matrice sableuse à silto-argileuse. La répartition spatio-temporelle des différents faciès au sein d'une même unité et dans les différentes unités morpho-sédimentaires de ce géo-système fluviatile est liée aux conditions hydrodynamiques. Ces dernières dépendent étroitement des conditions topographiques, morphologiques, hydrologiques et écologiques. Cette importante sédimentation détritique est l'expression sédimentaire d'un déséquilibre important dans le bassin versant. Ce déséquilibre est lié à une régression importante du couvert forestier et une érosion importante notamment des sols qui ont été complètement décapés dans certaines zones. Cette régression du couvert forestier dans la partie amont du bassin versant est due à la conjugaison d'une aridification du climat actuel et 
une accentuation de l'action anthropique ([9], [10], [11] et [12]). Cette action s'est manifestée par la régression sévère du couvert forestier sous l'effet du surpâturage, les coupe de bois et l'extension de l'agriculture au dépend des zones forestières.

Actuellement cette action anthropique, devenue plus importante, s'exprime par une accentuation $\mathrm{du}$ surpâturage, une dégradation du couver végétal et une ouverture des carrières pour l'extraction de sels à partir des argiles salifères du Tria et des sables et granulats à partir des terrains carbonatés jurassiques et les alluvions actuelles du lit mineur. Ces actions s'expriment par une accentuation du détritisme et un dysfonctionnement morphodynamique et écologique du géo-système fluviatile de l’Oued Beht moyen.

\section{Calage stratigraphique et taux de sédimentation}

Des observations de terrains dans le tronçon supérieur du Moyen Beht, ont montré une accumulation importante de décharges détritiques dans un cône de déjection sous un pont qui a été construit, à travers une chaâbat, en 2008. L'épaisseur de ce dépôt récent, de type débris flow, est de $1,60 \mathrm{~m}$; ce qui correspond à un taux de sédimentation moyen de $30 \mathrm{~cm} / \mathrm{an}$.

D'autres observations, effectuées toujours dans le même tronçon mais cette fois-ci en aval du site précédent, ont montré l'existence d'une partie d'une canalisation en béton armé dans un dépôt de plaine d'inondation. Cette plaine, qui se présente sous forme d'un replat bien développé de part et d'autre du lit mineur actuel méandriforme, présente un commandement d'environ $3 \mathrm{~m}$. Les témoins fournis par un agriculteur, ayant travaillé dans une ferme, qui appartenait à un colon français, ont rapporté que cette canalisation, actuellement située sous une tranche de sédiments fins sablo-silto-argileux de 3m d'épaisseur, a été construite en 1950. L'installation de cette canalisation a été faite pour effectuer une dérivation des eaux d'irrigation depuis le lit de l'Oued, qui est actuellement abandonnée, vers la rive gauche. D'après ces données, il sort clairement que cette plaine d'inondation de 2,5 à $3 \mathrm{~m}$ de côte relative, qui a été considérée, dans beaucoup de vallées marocaines, par plusieurs auteurs ([13], [14], [15], [16], [17] et [18]) comme une terrasse d'âge Rharbien ou Holocène, est très récente et remonte au $19-20^{\text {ème }}$ siècle. Le taux de sédimentation moyen calculé dans ces dépôts fin de plaine d'inondation est de $30 \mathrm{~cm} / \mathrm{an}$.

\section{CONCLUSION}

L'étude morpho-structurale de la vallée de l'Oued Beht moyen a permis de distinguer trois grands tronçons: deux tronçons larges, creusé chacun dans une dépression synclinale, que sépare un tronçon étroit et plus profond, incisé dans un dôme anticlinal. Les deux dépressions synclinales sont formées de terrains tendres et friables. Ces terrains sont composés d'argiles gypso-salifères et de basaltes altérés d'âge triasique que surmontent des grès friables et des marnes du Miocène supérieur. Le dôme anticlinal, d'origine tectonodiapirique, est formé de terrains triasiques qu'arment des carbonates durs du Jurassique. Ces terrains du substrat sont affectés par un réseau de failles qui ont guidé la configuration du réseau hydrographique actuel.

Dans les deux tronçons supérieur et inférieur de la vallée incisée dans les deux dépressions synclinales à terrains tendres et friables, les styles fluviaux sont méandriformes, avec des méandres libres et une tendance locale au tressage où vers des styles droit à faiblement sinueux, liée au rétrécissement du lit mineur et/ou à une accentuation de sa pente longitudinale. Dans ces tronçons, les unités morpho-sédimentaires les plus développées sont les barres, surtout de méandres, les plaines d'inondation, les méandres et les chenaux abandonnés. Localement se développent des levées naturelles et des cônes de déjection.

Dans le tronçon de la vallée creusée dans le dôme anticlinal, à cœur triasique et à armature carbonatée jurassique, le style est de type droit à faiblement sinueux à travers les deux flancs, méridional et septentrional de ce dôme et méandriformes, avec des méandres encaissés, à travers sa voûte. Dans ce tronçon, les unités morphosédimentaires les plus développées sont les barres longitudinales, les barres latérales, les barres des méandres encaissées, les levées naturelles, les cônes d'éboulis, qui jalonnent les bas des versants à corniche carbonatée, et les cônes de déjection qui deviennent relativement plus étendus. Dans ce tronçon les plaines d'inondation deviennent étroites voire même absentes dans les zones où la vallée devient plus rétrécie et profonde.

Les dépôts, presque entièrement détritiques, se répartissent, du point de vue textural, en deux types principaux : les dépôts de texture caillouteuse et sableuse et les dépôts de texture silto-argileuse. Les premiers se répartissent dans les fonds des chenaux actifs, les barres, les cônes de déjection et les cônes d'éboulis. Les seconds occupent les plaines d'inondation, les méandres et les chenaux abandonnés. Les dépôts des levées 
naturelles sont caractérisés par une texture sablo-silteuse. Les dépôts de texture caillouteuse sont mis en place par des écoulements fortement turbulents en périodes de crues. Les dépôts de texture silto-argileuse sont déposés par décantation lors des débordements des eaux sur la plaine d'inondation, les méandres et les chenaux abandonnés. La mise en place des dépôts de texture sablo-silteuse est effectuée par écoulements de débordements de turbulence moyenne. Les dépôts des cônes d'éboulis et d'épandages sont mis en place par éboulement des parois rocheuses carbonatées. Ces éboulements sont favorisés par la fracturation des roches et la cryoclastie. Notons que le remaniement de ces dépôts, en périodes de pluies orageuses, contribuent à l'engraissement de ceux des cônes de déjection.

De point de vue environnemental, l'importance des dépôts détritiques et du taux de la sédimentation actuelle est le résultat d'une importante régression du couvert forestier sous les effets conjugués d'une tendance de l'aridification du climat actuel et surtout d'une accentuation de l'action anthropique dans le bassin versant. Cette action s'exprime actuellement dans le tronçon supérieur de la vallée du Moyen Beht par une importante extraction de sables et de granulats à partir des dépôts des barres surtout de méandre ce qui conduit au dysfonctionnement morpho-dynamique, sédimentaire et écologique de ce géo-système fluviatile.

\section{REFERENCES}

[1] J. Burger, R. Dardel, E. Dutrieux, J. Jacquemont \& R. Naif, Carte géologique régulière du Maroc au 1:100.000eme: Meknès nord, Feuille levée et édifiée par la Société Chérifiènne des Pétroles, Notes et mémoires du Service géologique du Maroc, 111, 1951.

[2] Cirac P., Le bassin sud-rifain occidental au Néogène supérieur. Évolution de la dynamique sédimentaire et de la paléogéographie au cours d'une phase de comblement. Thèse de Doctorat d'État, Université Bordeaux $1, \mathrm{n}^{\circ} 837$ et Mémoire de l'Institut de Géologie du Bassin d'Aquitaine, Bordeaux, 21, 1987, 271p.

[3] Bavard, J.P. \& Petit, F., Les cours d'eau, dynamique du système fluvial, (Armand Colin ed., Paris, 1997), 221p.

[4] Taous, A., Géomorphodynamique fluviale. Processus morphosédimentaires, ajustements spatio-temporels, paléoenvironnements et mutations récentes des eaux fluviaux, Publications de la Faculté des Lettres et des Sciences Humaines Sais-Fès, Série "Thèses et Monographie », 11, 2005.

[5] Bull, W.B., Relations of alluvial-fan size and slope to drainage-basin size and lithology in western Frenso Country, California, U.S. Geol. Surv. Prof. Pap., 450-B, 1962, 51-53.

[6] Hooke, R. Le B., Steady-state relation-ships on arid region alluvial fans in closed basins, Amer. J. Sci., 266, 1968, 609-629.

[7] Rackoki, A. H., Alluvial fans. An Attempt at an empirical approach, (Wiley, Chichester, 1981), 161p.

[8] Herail, G., Les cônes de déjection : formes et sediments, Bull. Centre Rech. Explor. Prod. Elf-Aquitaine, Pau, 8 (1), 1984, 135-150, Notes. Ser. Géol. Maroc, 36 (264), 1984, 69-79.

[9] Gourari, L., Etude hydrochimique, morphologique, lithostratigraphique, sédimentologique et pétrographique des dépôts travertino-détritique actuels et plio-quaternaire du bassin kardtique de l'Oued Aggai (Causse de Sefrou, Moyen Atlas,Maroc), Thèse d'Etat, Université Sidi Mohamed Ben Abdellah, 2001, 408p.

[10] Tribak, A., L'érosion hydrique en moyenne montagne du Prérif oriental (Maroc) : étude des agents et des processus d'érosion dans une zone de marnes tertiaires, Thèse D'Etat, Université Chouaib Doukkali, El Jadida, 2000, 351p.

[11] Al Karkouri Jamal, Dégradation du milieu naturel dans le bassin versant de Beni Boufrah (Rif central-Maroc) analyse des facteurs et des processus, essais de quantification et de modélisation spatiale, Thèse d'Etat, Université Mohammed V, Rabat, 2003, 392p.

[12] Laabidi, A., Gourari, L., El Hmaidi, A., Aarab, M. \& Gretaa, M., Caractérisation géomorphologique, lithostratigraphique et sédimentologique des dépôts quaternaires de l'Oued Bou Salloum (Haute Moulouya, Maroc). International Journal of Engineering Research and Development, 10, 4, 2014, 7-19.

[13] Beaudet, G., Le Quaternaire marocain : état des etudes, Rev. géogr. Maroc, 22, 1971, 3-56.

[14] Laouina, A., Observations géomorphologiques dans la région du moyen Sebou en amont de Fès, Rev. Géogr. Maroc, 23-24, 1974, 95-123.

[15] Martin, J., Le moyen Atlas Central. Etude Géomorphologique, Notes et Mém. Serv. Geol. Maroc, 258 bis, 1981, 445 p.

[16] Dutour, A., Etude géomorphologique dela partie occidentale de la Haute Moulouya, Maroc, Thèse de $3^{\text {ème }}$ cycle, Université de Poitiers, France, 1983, 275p.

[17] Ouadia, M., Les formations philo-quaternaires dans le domaine mésétien occidental du Maroc entre Casablanca et Safi, Géomorphologie, sédimentologie, paléoenvironments quaternaires et évolution actuelle, Thèse d'Etat, Univ. Mohammed V, Rabat, 1998, 319p.

[18] Dridri A., Les formations fluviatiles quaternaire des oueds inaouene et moyen Sebou: Analyse lithologique, sédimentologique et morphotructurale, Thèse d'Etat, Université Sidi Mohamed Ben Abdellah, 2004, 308p. 